\title{
A case-based approach to UTI imaging - making sense of the guidelines
}

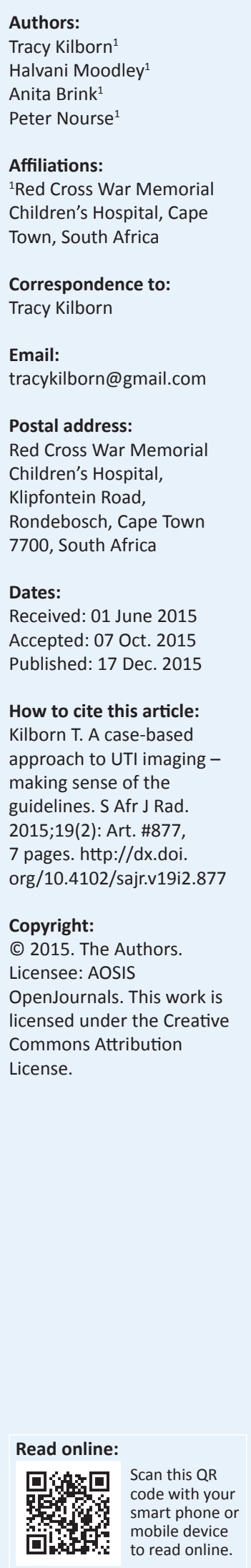

Urinary tract infections (UTIs) are common amongst children and invariably result in imaging tests to look for correctable causes that may predispose the child to infection. The objective of imaging is to identify those children at risk of long-term renal damage. The ideal imaging algorithm is extensively debated in the literature owing to the lack of evidence-based data, evolving theories on the pathophysiology of UTI and vesicoureteric reflux (VUR). The present article provides a case-based approach to the imaging of UTIs and proposes guidelines relevant to the South African setting.

\section{Introduction}

Approximately $7 \%-8 \%$ of girls and $2 \%$ of boys have a urinary tract infection (UTI) within the first 8 years of life, with the highest incidence within the first year in both sexes. ${ }^{1}$ Historic management of UTI has been based on the premise that recurrent UTIs, particularly with vesicoureteric reflux (VUR), increase the risk of chronic kidney disease, hypertension and ultimately end-stage renal disease. Guidelines therefore focus on aggressive treatment and extensive imaging studies to detect obstructive malformations, VUR and renal scarring. ${ }^{2,3}$

The National Institute for Health and Clinical Excellence (NICE) altered guidelines in the UK in 2007 and the AAP (American Academy of Pediatrics) in 2011, both of which recommended radically reducing the use of imaging, particularly fluoroscopy and nuclear medicine, in the setting of a first typical UTI. ${ }^{4,5}$ NICE in addition no longer recommends any imaging in a first UTI unless atypical (non-Escherichia coli organism, poor urine flow, abdominal mass, septicaemia, raised serum creatinine or failure to respond to antibiotics). ${ }^{6}$

The less aggressive approaches are finding favour as it is recognised that medical radiation exposure is not without risks and because of the uncertainty of benefits to the patient of identifying low-grade reflux and minor parenchymal scars. ${ }^{3}$ The actual relationship between VUR and renal scarring is poorly understood. Some relationship does exist; however, one does not require reflux to develop a scar nor does one always develop scarring with a reflux-related UTI. ${ }^{7,8}$ In some cases, renal damage previously attributed to acquired scarring post pyelonephritis or VUR is now recognised as being more likely the sequela of renal dysplasia. ${ }^{3,9}$ Also, a child with structurally normal kidneys is not at significant risk of developing chronic kidney disease because of UTIs. ${ }^{9}$

Another risk factor for scarring that receives less attention in the radiology literature is the presence of bladder and bowel dysfunction known as dysfunctional elimination (DE). The propensity for scarring is diminished when DE is effectively treated; therefore, regardless of the type of radiographic evaluation, the most important intervention may be to identify those children with DE and treat it. ${ }^{7}$ 


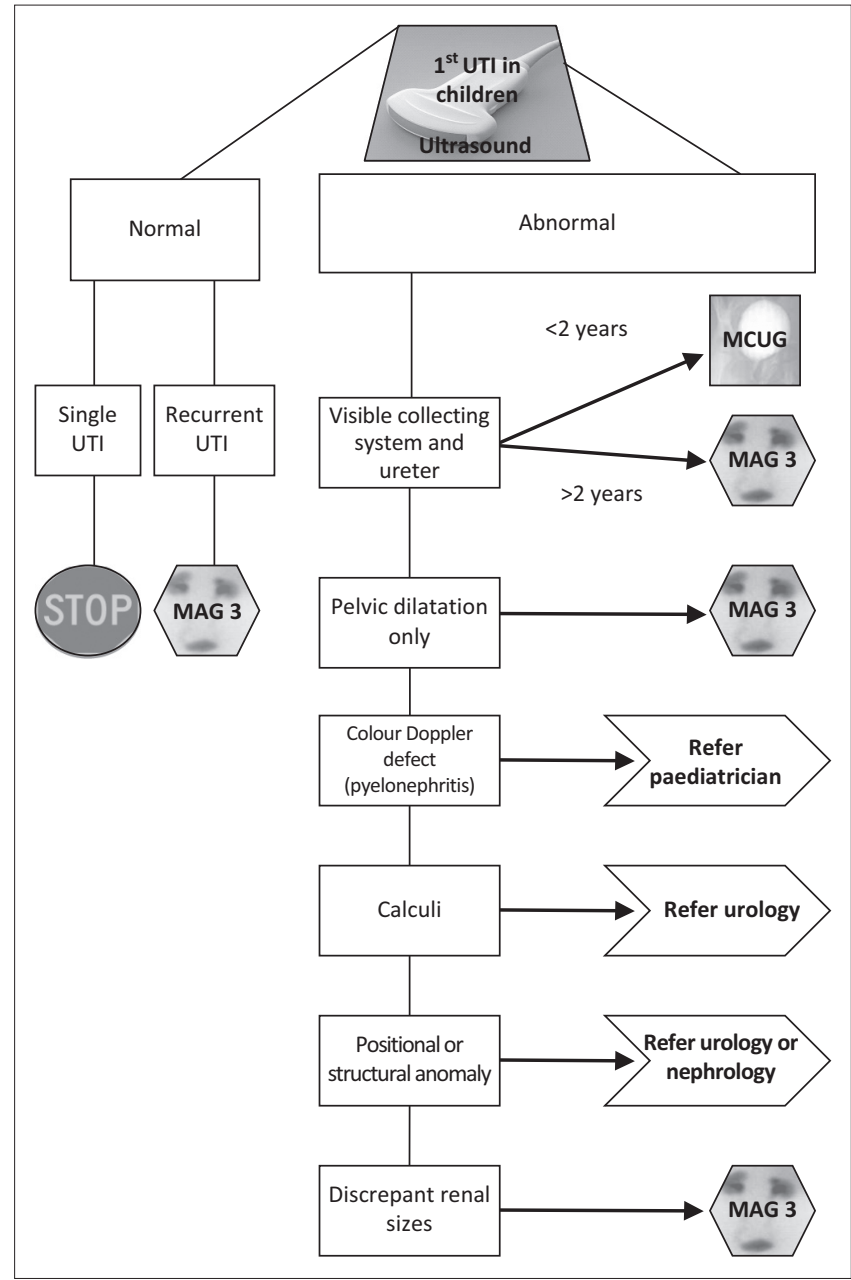

FIGURE 1: Suggested algorithm for management of paediatric UTI.

\section{Case-based approach to UTI}

\section{Case 1}

We recommend in our setting that all children presenting with a UTI undergo ultrasound.

Document renal length supine (Figure 2a) and/or prone (Figure 2b), and a look at vascularity (Figure 2c). Kidney sizes must be compared with age-related normal values at the time of scanning and these same norms should be used at follow-up. The anteroposterior diameter of the renal pelvis should always be measured at the hilum on the transverse view (Figure 2d; arrow). If normal (as in this case) and a firsttime UTI, the patient would require no further imaging.

\section{Ultrasound}

Ultrasound should remain part of the evaluation of first-time UTI as it provides a gross anatomic evaluation that is noninvasive and does not use ionising radiation. It can assist in depicting structural and positional anomalies, differential renal size, hydronephrosis, dilated ureters and bladder abnormalities. ${ }^{10}$ Despite the AAP stating that the diagnostic yield from ultrasound is low, as only $1 \%-2 \%$ of children will have actionable findings, it remains relevant in the South African setting. With poor access to antenatal scanning,
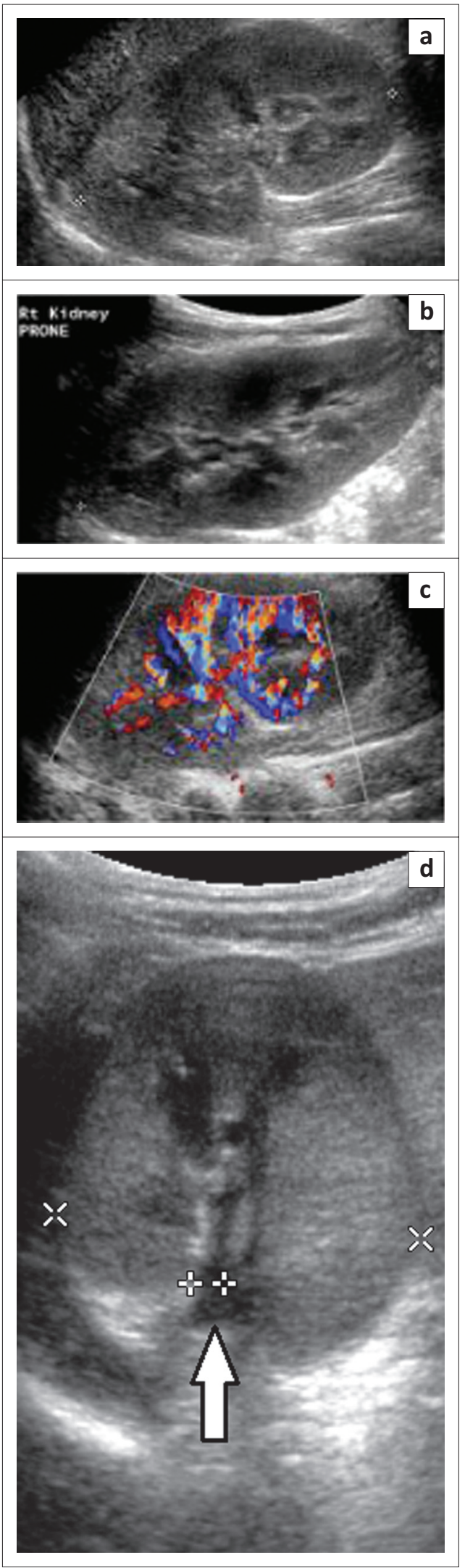

FIGURE 2: Ultrasound images referred to in the text.

abnormalities that would normally be identified (posterior urethral valve, pelviureteric junction obstruction and severe VUR) may present postnatally with a UTI. Ultrasound is limited by its inability to provide a quantitative assessment of 
BOX 1: What needs to be documented on a sonogram ${ }^{11}$

- Renal position
- Renal movement during scanning and the presence of tenderness
- Longitudinal renal sizes (compare with age-related norms)
- Echogenicity and corticomedullary differentiation
- Scars/focal lesions (prone position aids visualisation as does a linear probe in
- Symments)
perfusion which can be seen in pyelonephritis)
- Transverse anteroposterior measurement of the renal pelvis
- Measure ureter if visible and document if proximal, mid and/or distal
- Bladder wall thickness and volume pre and post micturition
- Ureterocoele
- Posterior urethra if dilated

renal function, has a sensitivity and specificity of $40 \%$ and $76 \%$ respectively for VUR, and is poor at demonstrating renal scars. ${ }^{9}$

Although widely available, the quality of the study is dependent on the skill of the technician, and therefore strict guidelines should be followed with regards to technique, particularly in paediatric patients. ${ }^{11}$ Despite the limitations, it is often comforting to the family and referring clinician to have a safe, non-invasive examination that grossly reveals the condition of the kidneys.

\section{Case 2}

\section{One-week-old boy with a UTI}
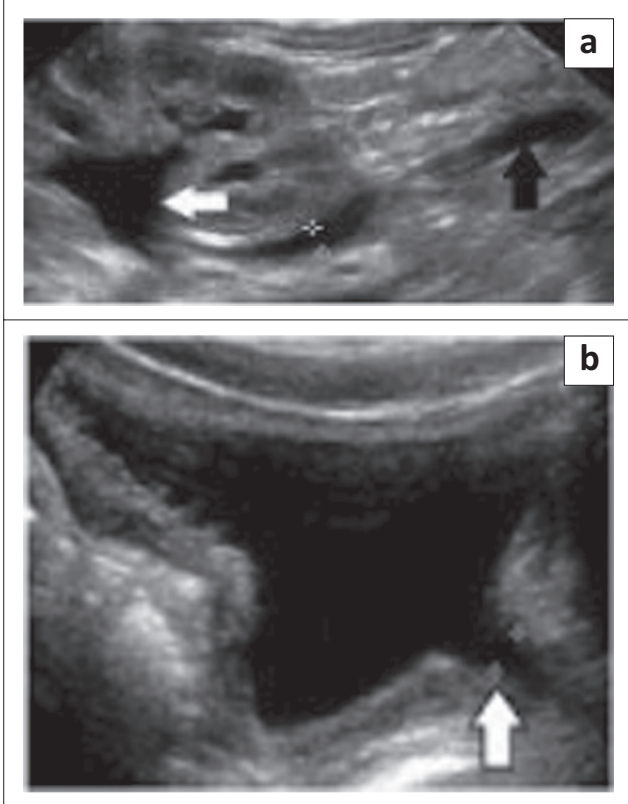

FIGURE 3: One-week-old male infant with UTI. Longitudinal ultrasound (a) shows dilated renal pelvis (white arrow) and a dilated ureter (black arrow) Longitudinal (b) and transverse(c) views of the bladder show wall thickening and a distended posterior urethra (white arrows). (d) The marked bladder thickening (white line) is better demonstrated on this transverse image post micturition. These findings suggest a posterior urethral valve and the patient requires an MCUG. (e) Frame grab image from MCUG confirms dilated posterior urethra (white arrow) and bilateral grade 5 VUR (black arrows).

MCUG, micturating cystourethrogram; VUR, vesicoureteric reflux.
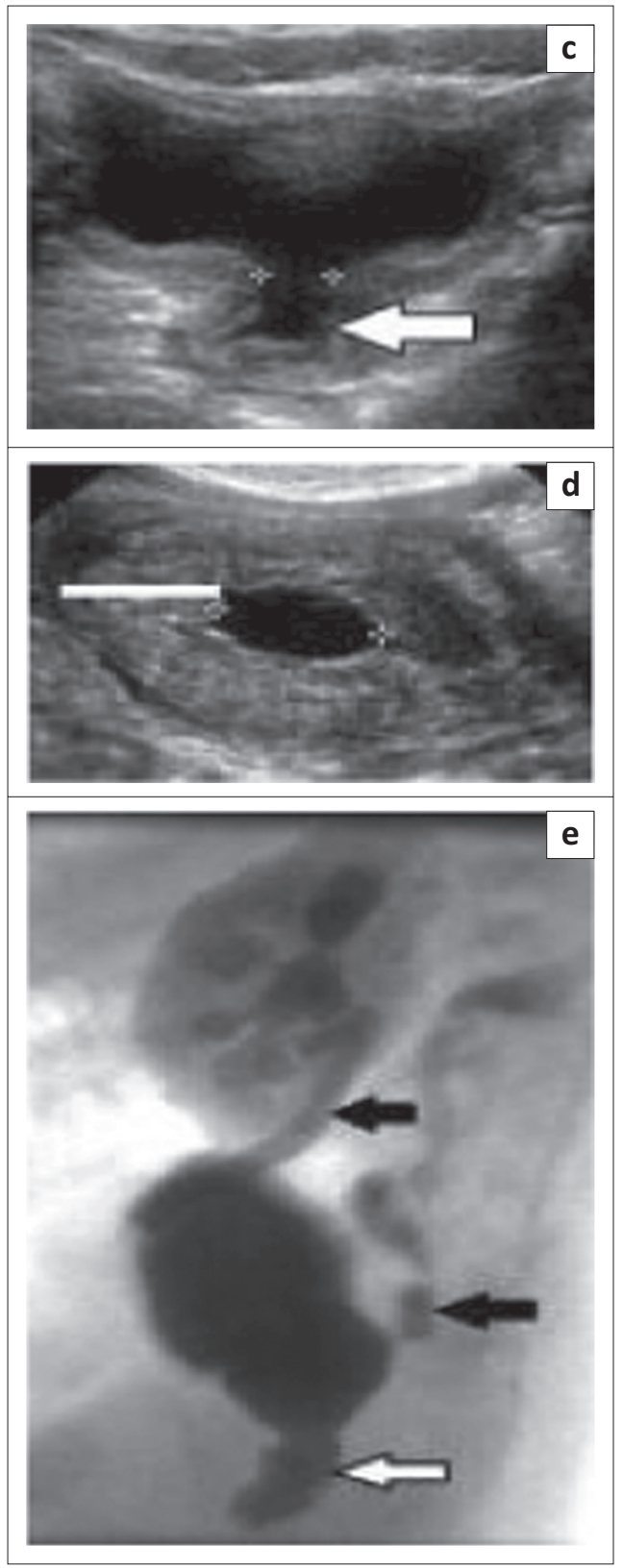


\section{Case 3}

\section{Eighteen-month-old with UTI}
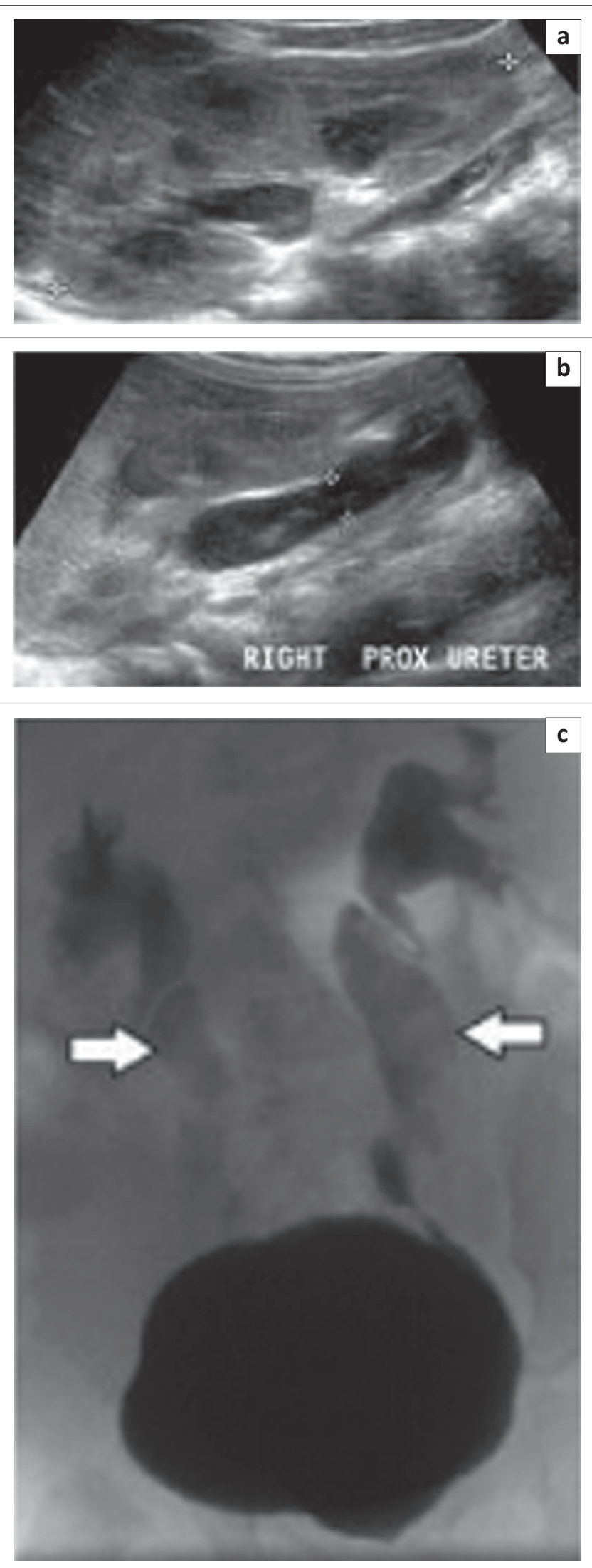

FIGURE 4: Longitudinal ultrasound of the kidney (a) and ureter (b) shows dilated pelvicalyceal system and ureter containing debris In a child under 2 years with dilatation of the pelvicalyceal system and ureter, MCUG is indicated. Frame grab image (4c) from MCUG confirming bilateral VUR (white arrows).

\section{Micturating cystourethrogram (MCUG)}

As a result of the controversy surrounding reflux and the attempt to limit exposure to ionising radiation, most of the discussion around UTI imaging has centred on the rationalised use of this test. Using modern grid-controlled variable rate pulsed fluoroscopy with last-frame grab reduces the dose to an eighth when compared with continuous screening. Nevertheless, the effective dose still remains higher than radionuclide cystography. ${ }^{10,12}$

MCUG should only be performed in the setting of an abnormal ultrasound (calyceal, pelvic and ureteric dilatation or scarring) to look for VUR in children under the age of 2 years. MCUG requires catheterisation that may be distressing for the patient and the parent. The advantages are the standardised international grading system for VUR and anatomical detail, particularly visualisation of the urethra in boys. ${ }^{10}$

\section{BOX 2: How to perform a MCUG ${ }^{13,14}$}

Tips
- Catheterise using an aseptic technique.
- Do urine dipstick analysis. If positive for infection, liaise with clinician about
re-scheduling examination if child is not on antibiotics and keep sample for
laboratory testing.
- Set up for pulsed fluoroscopy at 3 frames per second or less.
- Use last frame image grab rather than a full exposure.
- Bladder should be filled either by gravity or syringe. If using syringe, use gentle
pressure and do not exceed recommended volume for age. Expected volume =
([age in years +2$] \times 30) .{ }^{15}$
- Use warm, low osmolar contrast medium.
- Cone images to see bladder.
- Frame grab image of early filling view to look for ureterocoeles (Figure 4 ).
- In girls, leave patient supine and fill bladder until micturition.
- In boys, fill bladder in the oblique position so that the urethra is in profile.
- Frame grab images of micturition - there is no need to remove the catheter or
to do bilateral oblique projections as grade 1 reflux is not treated.
- Repeat bladder filling and micturition for a second time in infants.
- Record image of renal beds.
- Describe whether reflux was seen on passive filling and/or active micturition
and what grade is identified.

\section{Case 4}

\section{One-year-old with UT}

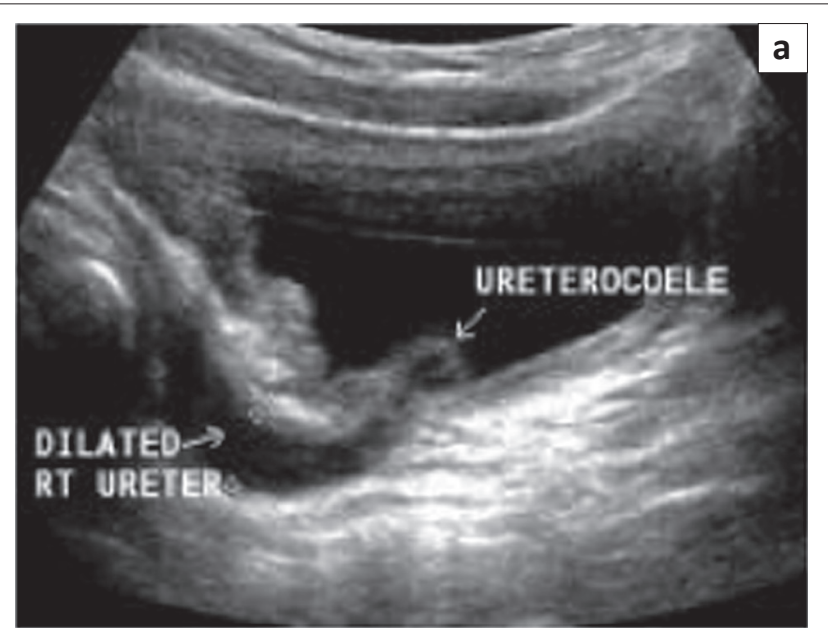

FIGURE 5: Longitudinal ultrasound (a) of the bladder shows dilated distal ureter and ureterocoele.

Figure 5 continues on the next page $\rightarrow$ 

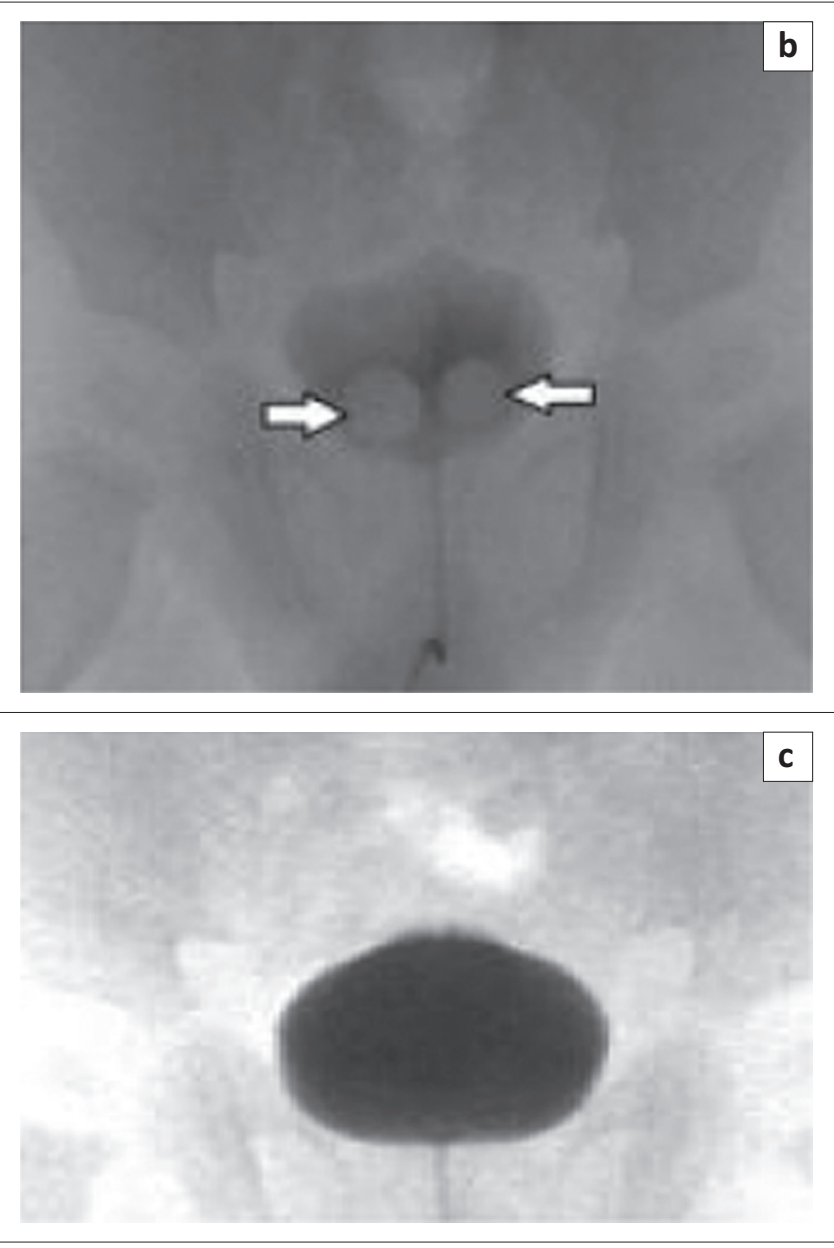

FIGURE 5 (Continues...): Longitudinal ultrasound (a) of the bladder shows dilated distal ureter and ureterocoele. Frame grab image (b) of early filling view on MCUG shows bilateral ureterocoeles (white arrows). Note how these are obscured in the full bladder view of the same patient (c).

\section{Case 5}

\section{Six-month-old boy with UTI}

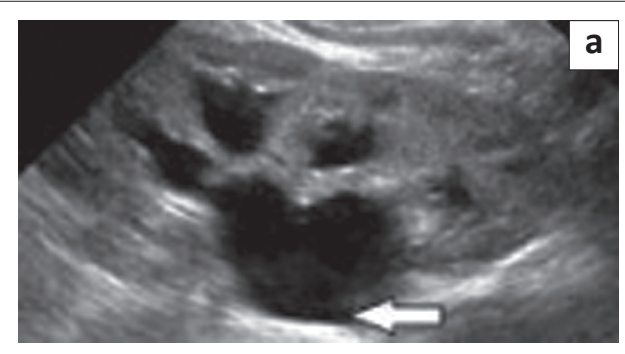

FIGURE 6: Longitudinal view (a) of the kidney shows hydronephrosis and dilated calyces. Transverse view (b) demonstrates where to measure the renal pelvis. No dilatation of the mid or distal ureter was seen (the proximal ureter may be seen in a PUJ obstruction but not the mid or distal ureter) and the bladder was normal. The patient has features of a pelviureteric junction obstruction and requires a ${ }^{99 m}$ Tc MAG3 study. Summated images (c) from the ${ }^{99 m}$ Tc MAG3 study show a normal left kidney - the corresponding renogram/activity curve (red) rises until 20 minutes with a normal decrease in amplitude after furosemide administration. The right kidney (outlined in green) appears larger than the left with a photopaenic area in the region of the pelvis. The corresponding activity curve (green) rises for the duration of the study with no response to furosemide. These findings confirm pelviureteric junction obstruction of the right kidney.
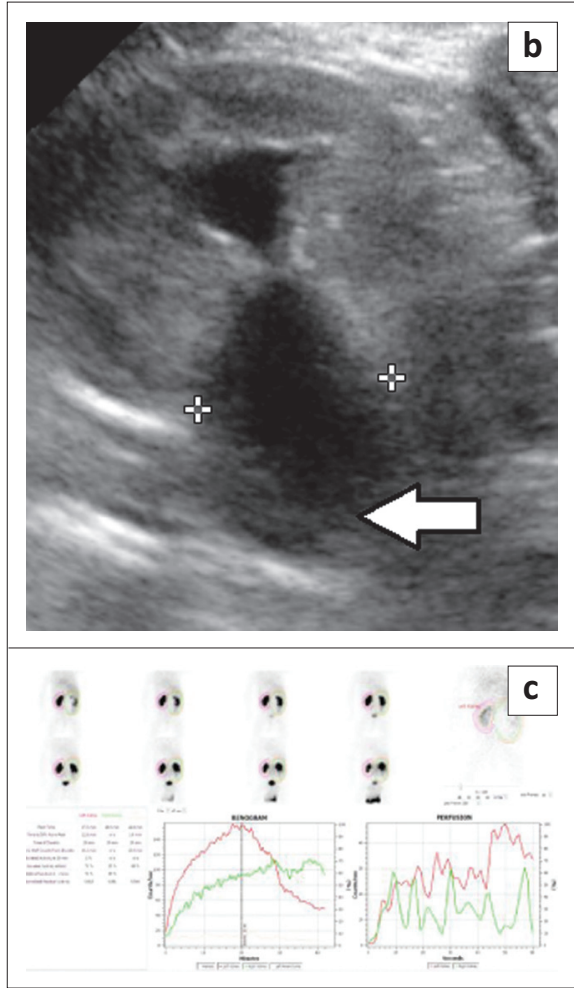

FIGURE 6 (Continues...): Longitudinal view (a) of the kidney shows hydronephrosis and dilated calyces. Transverse view (b) demonstrates where to measure the renal pelvis. No dilatation of the mid or distal ureter was seen (the proximal ureter may be seen in a PUJ obstruction but not the mid or distal ureter) and the bladder was normal. The patient has features of a pelviureteric junction obstruction and requires a ${ }^{99 \mathrm{~m} T c}$ MAG3 study. Summated images (c) from the ${ }^{99 \mathrm{~m} T c}$ MAG3 study show a normal left kidney - the corresponding renogram/activity curve (red) rises until 20 minutes with a normal decrease in amplitude after furosemide administration. The right kidney (outlined in green) appears larger than the left, with a photopaenic area in the region of the pelvis. The corresponding activity curve (green) rises for the duration of the study with no response to furosemide. These findings confirm pelviureteric junction obstruction of the right kidney.

\section{Case 6}

Ten-year-old with bilateral hydronephrosis and recurrent UTIs

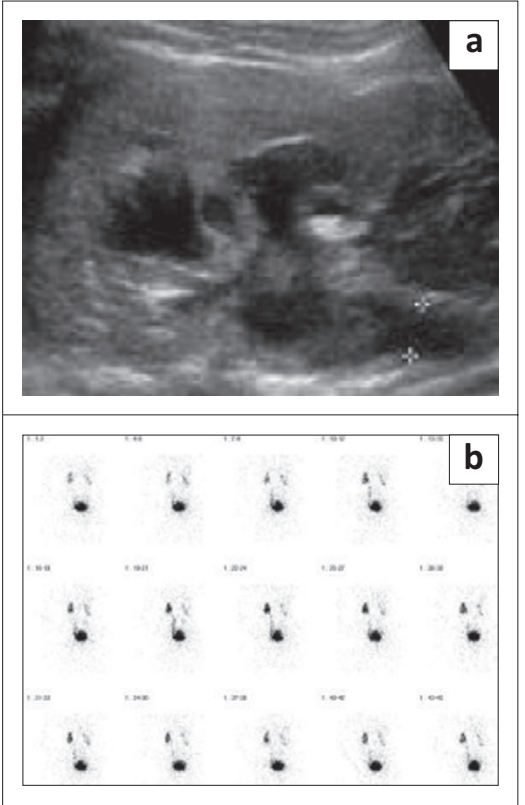

FIGURE 7: Longitudinal ultrasound image (a) demonstrates hydronephrosis ${ }^{99 m}$ Tc MAG3 indirect cystogram (b) demonstrates VUR into the left kidney from frames 7-9. The increase in activity in the left kidney is best appreciated when comparing frames 4-6 with frames 19-21. 


\section{Case 7}

Three-year-old with recurrent UTIs and normal ultrasound study (not shown)

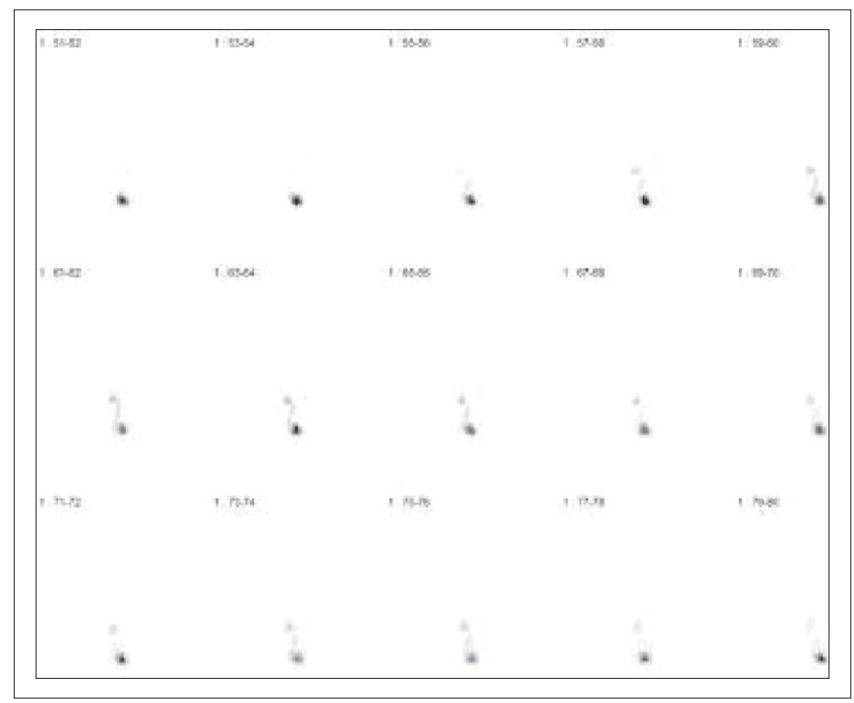

FIGURE 8: The ${ }^{99 m} \mathrm{mc}$ MAG3 indirect cystogram demonstrates left VUR from frames 55-56 onwards.

\section{Nuclear medicine in the investigation of UTI ${ }^{99 m}$ Tc-MAG3 versus ${ }^{99 m}$ Tc-DMSA}

Technetium-99m dimercaptosuccinic acid ( ${ }^{99 \mathrm{~m}} \mathrm{Tc}$ DMSA) is actively taken up by the proximal renal tubules and is regarded as the gold standard for the assessment of cortical defects. The long residence time of ${ }^{99 m}$ DMSA within the cortex leading to high radiation doses to paediatric patients has been a cause of concern. ${ }^{16}$

Technetium-99m mercaptoacetyltriglycine ( ${ }^{99 m}$ Tc MAG3) is a renal tubular agent. It provides information about cortical uptake, transit and drainage of tracer from each kidney. ${ }^{17}$ The tracer is rapidly excreted, and therefore there is a significant reduction in radiation dose $(0.3 \mathrm{mSv}$ for MAG3 as opposed to $1 \mathrm{mSv}$ for DMSA). ${ }^{17}$

${ }^{99 m}$ Tc-MAG3 is mainly used to evaluate hydronephrosis and renal drainage. Evaluation of the 1-2 minute image also allows the detection of cortical defects. Studies have shown that clear cortical defects seen on ${ }^{99 m}$ TC DMSA were also detected on ${ }^{99 \mathrm{~m}} \mathrm{Tc}$ MAG3 with sensitivity and specificity recorded as $88 \%-89 \%$ and $88 \%-100 \%$ respectively. ${ }^{16,17,18}$

We therefore recommend ${ }^{99 \mathrm{~m} T c-M A G 3}$ in children as it provides adequate estimation of the renal function, can evaluate for scars, provide information on drainage and be used to evaluate VUR in older children.

\section{Radionuclide cystography (RNC)}

In children who are toilet trained and have normal renal function, the indirect radionuclide cystography is an elegant investigation. At the end of a normal ${ }^{99 \mathrm{~m} T c}$ MAG3 renogram, most of the injected activity is in the bladder, and the activity from the kidneys has cleared. The child then voids in front of an upright camera, and VUR and bladder emptying can be evaluated. The advantage is that bladder catheterisation is not required and the radiation dose is less than that of an MCUG. In addition, the preceding renogram provides additional information about renal function and cortical defects. ${ }^{10}$ The disadvantage is reduced anatomic resolution and no imaging of the urethra. ${ }^{19} \mathrm{~A}$ RNC study should be considered in girls as urethral pathology is less common, in boys older than 2 years in whom posterior urethral valves are not suspected, and in follow-up studies assessing for resolution of VUR.

Direct RNC requires urethral catheterisation and direct installation of tracer. It is more sensitive than MCUG in detecting reflux at a reduced dose; however, it is not widely used as it lacks high spatial resolution and does not have the advantage of the functional assessment provided by the indirect study. ${ }^{20}$

\section{Emerging techniques Contrast-enhanced voiding ultrasonography (ceVUS)}

Sonographic assessment for VUR using installation of an intravesical contrast agent via catheter has gained popularity over the last decade, given the possibility of avoiding radiation. Sensitivity and specificity for the detection of reflux are reported as $90 \%$ and $92 \%$ respectively. ${ }^{21}$ There are still a number of limitations that restrict its widespread use: the availability of the contrast agent and contrast modality scan mode on the machine; the cost is significantly higher than that of an MCUG or RNC; the procedure is long, lacks standardisation and depends on the experience of the operator $^{19,20}$; and it also still requires catheterisation of the bladder. The inadequate assessment of bladder morphology and lack of a panoramic view of the urinary tract are also reported to be disadvantageous. ${ }^{18}$ Urethrosonography during VUS has been reported but it is not widely practiced and urethral imaging is still largely done via MCUG. ${ }^{19,22}$

\section{Three-dimensional ultrasound (3D US)}

3D US improves the calculation of renal parenchymal volume and is particularly useful in irregularly shaped or hydronephrotic kidneys as the collecting system can be subtracted from the trace of the renal parenchymal volume. The rendered view gives images comparable to those of IVU and MRU. ${ }^{23}$

3D ultrasound-based cystoscopy allows visualisation of the internal surface of the bladder. ${ }^{24}$

\section{MRI}

Functional MR urography (MRU) is widely used in evaluation of the anatomy of the paediatric urinary tract. Dynamic contrast-enhanced MRU can provide functional evaluation 
of renal uptake, excretion and drainage. ${ }^{10}$ The spatial and contrast resolution have shown promise in differentiating between acquired renal damage and renal dysplasia. ${ }^{7}$

Interactive MRI for VUR (iMRVC) shows promise, providing results comparable to MCUG. ${ }^{25}$

The benefits of MRI and the lack of radiation remain offset by the sedation needed for the lengthy examinations, the cost of the procedure and lack of access to the study by the vast majority of patients undergoing investigation for UTI.

\section{Conclusion}

Imaging of paediatric UTI is complex and controversial with no universally accepted protocol. We have attempted to provide an approach relevant to our setting whilst taking into account the evolution in guidelines that seek to limit unnecessary intervention and minimise radiation exposure.

\section{Competing interests}

The author declares that she has no financial or personal relationships which may have inappropriately influenced her in writing this article.

\section{Authors' contributions}

T.K. (Red Cross War Memorial Children's Hospital) was responsible for the literature search. Document construction. Figure editing. H.M. (Red Cross War Memorial Children's Hospital) - Image collection. Schematics. Document editing. A.B. (Red Cross War Memorial Children's Hospital) - Image collection. Text editing. Fact checking. P.N. (Red Cross War Memorial Children's Hospital) - Literature review. Text editing. Fact checking.

\section{References}

1. Montini G, Tullus $K$, Hewitt $I$. Febrile urinary tract infections in children. N Engl J Med. 2011;365:239-250. PMID: 21774712, http://dx.doi.org/10.1056/ NEJMra1007755

2. Paintsil E. Update on recent guidelines for the management of urinary tract infections in children: The shifting paradigm. Curr Opin Pediatr. 2013;25:88-94. PMID: 23241875, http://dx.doi.org/10.1097/MOP.0b013e32835c14cc

3. La Scola C, De Mutiis C, Hewitt IK, et al. Different guidelines for imaging after first UTI in febrile infants: Yield, cost and radiation. Pediatrics. 2013;131:665-671. PMID: 23439905, http://dx.doi.org/10.1542/peds.2012-0164

4. Tullus K. What do the latest guidelines tell us about UTIs in children under 2 years of age. Pediatr Nephrol. 2012;27:509-511. PMID: 22203365, http://dx.doi. org/10.1007/s00467-011-2077-5

5. Roberts KB. Revised AAP guideline on UTI in febrile infants and young children. Am Fam Physician. 2012;86:940-947. PMID: 23157147.
6. Urinary tract infection in children: Diagnosis, treatment and long-term management. NICE clinical guideline 54. London: National Institute for Health and Clinical Excellence; 2007. c2007 [cited 2015 Apr 20]. Available from: http://www. nice.org.uk/nicemedia/live/11819/36032/36032.pdf

7. Prasad MM, Cheng EY. Radiographic evaluation of children with febrile urinary tract infection: Bottom-up, top-down, or none of the above? Adv Urol. 2012; Article ID 716739, 8 pages. http://dx.doi.org/10.1155/2012/716739

8. Moorthy I, Easty M, McHugh K, Ridout D, Biassoni L, Gordon I. The presence of vesicoureteric reflux does not identify a population at risk for renal scarring following a first urinary tract infection. Arch Dis Child. 2005;90:733-736. http:// following a first urinary tract infection.
dx.doi.org/10.1136/adc.2004.057604

9. Salo J, Ikaheimo R, Tapiainen T, Uhari M. Childhood urinary tract infections as a cause of chronic kidney disease. Pediatrics. 2011;128:840-847. http://dx.doi. org/10.1542/peds.2010-3520

10. Lim R. Vesicoureteral reflux and urinary tract infection: Evolving practices and current controversies in pediatric imaging AJR Am J Roentgenol. 2009;192: 1197-1208. PMID: 19380542, http://dx.doi.org/10.2214/AJR.08.2187

11. Govender N, Andronikou S, Goodier MD. Adequacy of pediatric renal tract ultrasound requests and reports in a general radiology department. Pediatr ultrasound requests and reports in a general radiology department. Pediatr
Radiol. 2012;42:188-195. PMID: 21997513, http://dx.doi.org/10.1007/s00247Radiol. 2012 $011-2259-0$

12. Ward VL, Strauss KJ, Barnewoldt CE, et al. Pediatric radiation exposure and effective dose reduction during voiding cystourethrography. Radiology. 2008;249: 1002-1009. PMID: 18941159, http://dx.doi.org/10.1148/radiol.2492062066

13. Agrawalla S, Pearce R, Goodman TR. How to perform the perfect voiding cystourethrogram. Pediatr Radiol. 2004;34:114-119. PMID: 14564427, http:// cystourethrogram. Pediatr Radiol. 2004
dx.doi.org/10.1007/s00247-003-1073-8

14. Goodman TR, Kilborn T, Pearce R. Warm or cold contrast medium in the micturating cystourethrogram (MCUG): Which is best? Clin Radiol. 2003:58:551-554. PMID: 12834639, http://dx.doi.org/10.1016/S0009-9260(03)00161-2

15. Zerin JM, Chen E, Ritchey ML, Bloom DA. Bladder capacity as measured at voiding cystourethrography in children: Relationship to toilet training and frequency cystourethrography in children: Relationship to toilet training and frequency
of micturition. Radiology. 1993:186:803-806. PMID: 8497634, http://dx.doi. of micturition. Radiology. 1993;186:80.1148/radiology.187.3.8497634
org/10

16. Othman S, Al-Hawas A, Al-Maqtari R. Renal cortical imaging in children: ${ }^{99 \mathrm{~m}} \mathrm{Tc}$ MAG3 versus ${ }^{99 m}$ Tc DMSA. Clin Nucl Med. 2012;37:351-355. PMID: 22391703, http:// dx.doi.org/10.1097/RLU.0b013e3182443f68

17. Ritchie G, Wilkinson AG, Prescott RJ. Comparison of differential renal function using technetium-99m mercaptoacetyltriglycine (MAG3) and technetium-99m dimercaptosuccinic acid (DMSA) renography in a pediatric population. Pediatr Radiol. 2008;38:857-862. PMID: 18551288, http://dx.doi.org/10.1007/s00247008-0908-8

18. Smokvina A, Grbac-Ivanković S, Girotto N, Dežulović MS, Saina G, Barković MM. The renal parenchyma evaluation: MAG3 vs. DMSA. Coll Antropol. 2005;29:649-654. PMID: 16417177.

19. Darge K. Voiding urosonography with ultrasound contrast agents for the diagnosis of vesicoureteric reflux in children. Comparison with radiological examinations. Pediat Radiol. 2008;38:54-63.

20. Kis E, Nyitrai A, Varkonyi I, et al. Voiding urosonography with second-generation contrast agent versus voiding cystourethrography. Pediatr Nephrol. 2010:25: 2289-2293. PMID: 20686902, http://dx.doi.org/10.1007/s00467-010-1618-7

21. Darge K, CEUS task force of the Society for Pediatric Radiology. Contrast-enhanced US (CEUS) in children: Ready for prime time in the United States. Pediatr Radiol. 2011;41:1486-1488. PMID: 21938505, http://dx.doi.org/10.1007/s00247-0112240-y

22. Duran C, Valera A, Alguersuari A, et al. Voiding urosonography: The study of the urethra is no longer a limitation of the technique. Pediatr Radiol. 2009:39:124-131. PMID: 19002449, http://dx.doi.org/10.1007/s00247-008-1050-3

23. Darge K, Grattan-Smith DJ, Riccabona M. Pediatric uroradiology: State of the art. Pediatr Radiol. 2011;41:82-91. PMID: 20407766, http://dx.doi.org/10.1007/ s00247-010-1644-4

24. Riccabonna M, Pilhatsch A, Haberlik A, Ring E. Three-dimensional ultrasoundbased virtual cystoscopy of the pediatric urinary bladder: A preliminary report on feasibility and potential value. J Ultrasound Med. 2008;27:1453-1459. PMID: 18809955.

25. Arthurs OJ, Edwards AD, Joubert I, Graves MJ, Set PA, Lomas DJ. Interactive magnetic resonance imaging for paediatric vesicoureteric reflux (VUR). Eur J Radiol. 2013;82:e112-e119. PMID: 23238363, http://dx.doi.org/10.1016/j. ejrad.2012.10.024 